\title{
ANNIHILATOR AND COMPLEMENTED BANACH*-ALGEBRAS
}

\author{
B. J. TOMIUK and PAK-KEN WONG
}

(Received 18 August 1969)

Communicated by E. Strzelecki

\section{Introduction}

The study of complemented Banach*-algebras taken up in [1] was confined mainly to $B^{*}$-algebras. In the present paper we extend this study to (right) complemented Banach*-algebras in which $x^{*} x=0$ implies $x=0$. We show that if $A$ is such an algebra then every closed two-sided ideal of $A$ is a ${ }^{*}$-ideal. Using this fact we obtain a structure theorem for $A$ which states that if $A$ is semi-simple then $A$ can be expressed as a topological direct sum of minimal closed two sided ideals each of which is a complemented Banach*-algebra. It follows that $A$ is an $A^{*-}$ algebra and is a dense subalgebra of a dual $B^{*}$-algebra $\mathfrak{A}$, which is determined uniquely up to *-isomorphism.

A Banach*-algebra $A$ is said to have the weak $\left(\beta_{k}\right)$ property if for every minimal left ideal $I$ of $A$ there exists a constant $k>0$ such that $\|x\|^{2} \leqq k\left\|x^{*} x\right\|$ for all $x \in I$. This concept is introduced in 5, where we also show its relation to annihilator properties in Banach*-algebras. An $A^{*}$-algebra which is a dense twosided ideal of a dual $B^{*}$-algebra has the weak $\left(\beta_{k}\right)$ property. A semi-simple complemented Banach*-algebra with the weak $\left(\beta_{k}\right)$ property is a dual $A^{*}$-algebra. In 6 we look at the weakly completely continuous $A^{*}$-algebras. Lemma 5.5 plays a prominent role in the development of 5 and 6 , as well as that of 7 . (In this context see [6] Lemmas 8 and 9.

In 7 we study dual $A^{*}$-algebras. We give several characterizations of duality for $A^{*}$-algebras, one of which is expressed in terms of (right) complementors. We show, in particular, that if $A$ is a dense two-sided ideal of a $B^{*}$-algebra then $A$ is dual if and only if it is complemented. In 8 we look at complementors induced by given complementors. More precisely, let $A$ be an $A^{*}$-algebra which is a dense subalgebra of a $B^{*}$-algebra $\mathfrak{A}$ and let $p$ be a complementor on $\mathfrak{A}$ and $q$ a complementor on $A$. We find conditions on $\mathfrak{A}, A$ and the complementors $p$ and $q$ such that: (a) The mapping $I \rightarrow \operatorname{cl}(I)^{\mathrm{p}} \cap A$ on the closed right ideals $I$ of $A$ is a complementor on $A$. (b) The mapping $R \rightarrow \operatorname{cl}\left((R \cap A)^{q}\right)$ on the closed right ideals $R$ of $\mathfrak{A}$ is a complementor on $\mathfrak{A}$.

In 9 we discuss an example of a complemented $A^{*}$-algebra. 


\section{Preliminaries}

Let $A$ be a complex Banach algebra and let $L_{r}$ be the set of all closed right ideals of $A$. Following [10], we shall say that $A$ is a right complemented Banach algebra if there exists a mapping $p: R \rightarrow R^{p}$ of $L_{r}$ into itself having the following properties:

$$
\begin{array}{lll}
\left(C_{1}\right) & R \cap R^{p}=(0) & \left(R \in L_{r}\right) ; \\
\left(C_{2}\right) & R+R^{p}=A & \left(R \in L_{r}\right) ; \\
\left(C_{3}\right) & \left(R^{p}\right)^{p}=R & \left(R \in L_{r}\right) ; \\
\left(C_{4}\right) & \text { if } R_{1} \subseteq R_{2} \text {, then } R_{2}^{p} \subseteq R_{1}^{p}\left(R_{1}, R_{2} \in L_{r}\right) .
\end{array}
$$

The mapping $p$ is called a right complementor on A. Analogously we define a left complemented Banach algebra and a left complementor. Thus a complex Banach algebra is left (right) complemented if and only if it has a left (right) complementor defined on it. A left and right complemented Banach algebra is called bicomplemented. We shall restrict our attention to right complemented Banach algebras. Therefore, unless mentioned otherwise, a complementor on a Banach algebra will always mean a right complementor and a complemented Banach algebra will always mean a right complemented Banach algebra. All Banach algebras and Banach spaces under consideration are over the complex field $C$.

For any set $S$ in a Banach algebra $A$, let $l(S)$ and $r(S)$ denote the left and right annihilators of $S$ respectively. A Banach algebra $A$ is called an annihilator algebra if $l(A)=r(A)=(0)$, and if for every proper closed right ideal $I$ and every proper closed left ideal $J, l(I) \neq(0)$ and $r(J) \neq(0)$. If, in addition, $r(l(I))=I$ and $l(r(J))=J$, then $A$ is called a dual algebra.

A Banach algebra $A$ is called simple if it is semi-simple and if $(0)$ and $A$ are the only closed two-sided ideals of $A$. An idempotent $e$ in a Banach algebra $A$ is said to be minimal if $e A e$ is a division algebra. In case $A$ is semi-simple, this is equivalent to saying that $A e(e A)$ is a minimal left (right) ideal of $A$.

A Banach algebra with an involution $x \rightarrow x^{*}$ is called a Banach*-algebra. A Banach*-algebra $A$ is called a $B^{*}$-algebra if the norm and the involution satisfy the condition $\left\|x^{*} x\right\|=\|x\|^{2}, x \in A$. If $A$ is a Banach*-algebra on which there is defined a second norm $|\cdot|$ which satisfies, in addition to the multiplicative condition $|x y| \leqq|x||y|$, the $B^{*}$-algebra condition $\left|x^{*} x\right|=|x|^{2}$, then $A$ is called an $A^{*}$-algebra. The norm $|\cdot|$ is called an auxiliary norm. Let $A$ be an $A^{*}$-algebra. Then $A$ is semi-simple, the involution in $A$ is continuous with respect to the given norm $\|\cdot\|$ and the auxiliary norm $|\cdot|$ and $|\cdot| \leqq \beta\|\cdot\|$ for a real constant $\beta$ (see [8] p. 187).

Let $H$ be a Hilbert space with inner product (,). If $x$ and $y$ are elements of $H$, then $x \otimes y$ will denote the operator on $H$ defined by the relation $(x \otimes y)(h)=$ 
$(h, y) x$ for all $h \in H$. Let $L(H)$ be the algebra of all continuous linear operators on $H$ into itself with the usual operator bound norm. $L C(H)$ will denote the subalgebra of $L(H)$ consisting of all compact operators on $H$.

Let $\left\{A_{\lambda}: \lambda \in \Lambda\right\}$ be a family of Banach algebras $A_{\lambda}$, and let $\left(\sum A_{\lambda}\right)_{0}$ be the set of all functions $f$ defined on $\Lambda$ such that $f(\lambda) \in A_{\lambda}$ for each $\lambda \in \Lambda$ and such that, for arbitrary $\varepsilon>0$, the set $\{\lambda:\|f(\lambda)\| \geqq \varepsilon\}$ is finite. It is easy to see that $\left(\sum A_{\lambda}\right)_{0}$ is closed under the usual operations of addition, multiplication and scalar multiplication for functions. $\left(\sum A_{\lambda}\right)_{0}$ is a Banach algebra under the norm $\|f\|=$ sup $\{\|f(\lambda)\|: \lambda \in \Lambda\}$. If each $A_{\lambda}$ is a $B^{*}$-algebra, then $\left(\sum A_{\lambda}\right)_{0}$ is also a $B^{*}$ algebra under the norm $\|f\|$ and the involution $f \rightarrow f^{*}$ given by $\left(f^{*}\right)(\lambda)=f(\lambda)^{* \lambda}$, where $* \lambda$ is the involution on $A_{\lambda} \cdot\left(\sum A_{\lambda}\right)_{0}$ is called the $B^{*}(\infty)$-sum of $A_{\lambda}$. If, in addition, $A_{\lambda}$ are dual, then $\left(\sum A_{\lambda}\right)_{0}$ is dual ([8], Theorem (4.10.25)).

Let $A$ be a dual $B^{*}$-algebra and $\left\{I_{\lambda}: \lambda \in \Lambda\right\}$ the family of all minimal closed two-sided ideals of $A$. Then $A$ is isometrically *-isomorphic to $\left(\sum I_{\lambda}\right)_{0}$. Since each $I_{\lambda}$ is isometrically ${ }^{*}$-isomorphic to $L C\left(H_{\lambda}\right)$, for some Hilbert space $H_{\lambda}$, we see that $A$ is isometrically *-isomorphic to $\left(\sum L C\left(H_{\lambda}\right)\right)_{0}$ (see [8] Chap. IV, $\S 10$ ). A $B^{*}$-algebra is dual if and only if it is complemented ([1] Theorem 3.6). We shall often use, without explicitly mentioning, the following fact about dual $B^{*}$-algebras: If $A$ is a dual $B^{*}$-algebra then the mapping $R \rightarrow l(R)^{*}$ on the set of all closed right ideals $R$ of $A$ is a complementor on $A$ (see [10] p. 652).

Let $X$ be a topological space and $S$ a subset of $X$. Then $\operatorname{cl}(S)$ will denote the closure of $S$ in $X$. The norm in a $B^{*}$-algebra will always be denoted by $|\cdot|$.

We shall need the following lemma:

Lemma 2.1. Let $A$ be a semi-simple Banach algebra with a dense socle. Then for every proper closed two-sided ideal $I$ of $A, l(I)=r(I) \neq(0)$. Moreover, every closed left (right) ideal of the algebra $I$ is also a closed left (right) ideal of $A$.

Proof. If $A$ is simple, the lemma is trivially true. So suppose $A$ is not simple. Since the socle is dense in $A$, there exists a minimal idempotent $e$ of $A$ such that $e \notin I$. Let $J$ be the closed two-sided ideal generated by $e$. By the proof of [2] Theorem $5, J$ is a minimal closed two-sided ideal of $A$. Since $e \notin I, I \cap J=(0)$ and so $J \subset l(I)$, which shows that $l(I) \neq(0)$. By the proof of [8] Lemma (2.8.10), we have that $l(I)=r(I)$ and that, if $R=\operatorname{cl}(I+l(I))$, then $l(R)=(0)$. Since every proper closed two-sided ideal of $A$ has a non-zero annihilator, we must have $R=A$. The second part of the lemma now follows from the proof of [8] Lemma (2.8.11).

\section{Annihilator complemented Banach algebras}

In this section, as well as in the rest of the paper, a complemented Banach algebra will always mean a right complemented Banach algebra.

Let $A$ be a complemented Banach algebra with a complementor $p$. We shall 
call an idempotent $e$ in $A$ a p-projection if $(e A)^{p}=\{x-e x: x \in A\}$. If ,moreover, $e$ is a minimal idempotent, we shall say that $e$ is a minimal p-projection. (In [10], a $p$-projection is called a left projection).

LEMMA 3.1. Let A be a semi-simple annihilator complemented Banach algebra with a complementor $p$. Then every non-zero right ideal I contains a minimal $p$ projection. Moreover, if $I$ is a closed non-zero right ideal and $\left\{e_{\alpha}\right\}$ is the family of minimal p-projections in $I$, then $I=\operatorname{cl}\left(\sum_{\alpha} e_{\alpha} A\right)$.

Proof. Let $R$ ba a minimal right ideal contained in $I$. Since $R^{p}$ is a maximal closed right ideal, by [2] Theorem $1, R^{p}$ is modular. The existence of a minimal $p$-projection in $I$ now follows from [10] Lemma 2 . To prove the second part of the lemma, suppose that $I \neq \operatorname{cl}\left(\sum_{\alpha} e_{\alpha} A\right)$; let $J=\operatorname{cl}\left(\sum_{\alpha} e_{\alpha} A\right)$. Then there exists $x \in I$ such that $x \notin J$. Write $x=x_{1}+x_{2}$ with $x_{1} \in J$ and $x_{2} \in J^{p}$. Then $0 \neq x_{2}=x-x_{1} \in I$ and so $I \cap J^{p} \neq(0)$. Hence there exists a minimal p-projection $e$ in $I \cap J^{p} \subset I$ which does not belong to $J$; a contradiction. Therefore $I=J$.

Combining Lemma 3.1 and [1] Lemma 2.1, we obtain the following result:

COROLlaRY 3.2. Let $A$ be an annihilator semi-simple complemented Banach algebra. Then every closed right ideal of $A$ is the intersection of maximal modular right ideals containing it.

THEOREM 3.3: Let A be a semi-simple complemented Banach algebra with a complementor $p$. Then the following statements are equivalent:

(i) $A$ is an annihilator algebra.

(ii) Every non-zero right ideal contains a minimal p-projection.

(iii) Every maximal closed right ideal is modular.

(iv) Every maximal closed right ideal has a non-zero left annihilator.

ProOF. (i) $\Rightarrow$ (ii). This follows from Lemma 3.1 .

(ii) $\Rightarrow$ (iii). Suppose (ii) holds and let $M$ be a maximal closed right ideal of $A$. Then $M^{p}$ is a minimal right ideal and hence $M^{p}=e A$, where $e$ is a minimal $p$-projection.

(iii) $\Rightarrow$ (iv). Let $M$ be a maximal closed right ideal. If $M$ is modular, [10] Lemma 2 shows that $M=\{x-e x: x \in A\}$, for some idempotent $e$, and hence $l(M) \neq(0)$.

(iv) $\Rightarrow$ (i). Let $I$ be a proper closed right ideal and $R$ a minimal right ideal contained in $I$ ([10] Corollary Theorem 1$)$. Then $R^{p}$ is a maximal closed right ideal and $I \subset R^{p}$. Hence if $l\left(R^{p}\right) \neq(0)$, then $l(I) \neq(0)$ and so, by [10] Theorem 8 , $A$ is an aniihilator algebra.

THEOREM 3.4. Let $A$ be an annihilator semi-simple complemented Banach algebra. Then every closed two-sided ideal of $A$ is an annihilator semi-simple complemented Banach algebra. 
Proof. Let $M$ be a closed two-sided ideal of $A$. Since, by [10] Lemma 1, $M^{p}=l(M)=r(M)$, every closed left (right) ideal of $M$ is a closed left (right) ideal of $A$; so that $M$ is semi-simple. Now $p_{M}: I \rightarrow I^{p_{M}}=I^{p} \cap M$ is a complementor on the closed right ideals of $M$. Hence if $I$ is a maximal closed right ideal of of $M$, then $I^{p_{M}}$ is a minimal right ideal of $M$ and also of $A$. Thus $\left(I^{p_{M}}\right)^{p}$ is a maximal closed right ideal of $A$ and therefore modular. But, by [10] Lemma 2,

$$
\left(I^{p_{M}}\right)^{p}=\{x-e x: x \in A\},
$$

where $e$ is an idempotent in $I^{p_{M}}$. Hence, since $I=\left(I^{p_{M}}\right)^{p} \cap M$,

$$
I=\{x-e x: x \in M\},
$$

i.e., $I$ is modular. Therefore, by Theorem $3.3, M$ is an annihilator algebra.

\section{Complemented Banach*-algebras}

Throughout this section, $p$ will denote the given complementor on the complemented Banach*-algebra $A$.

Lemma 4.1. Let $A$ be a semi-simple complemented Banach*-algebra. Then the involution in $A$ is continuous and hence $A$ is bicomplemented.

Proof. By [10] Lemma 5, the socle of $A$ is dense in $A$ and therefore, by [8] Corollary (2.5.8), $A$ has a unique norm topology. Hence the involution is continuous and consequently the mapping

$$
q: J \rightarrow J^{q}=\left(\left(J^{*}\right)^{p}\right)^{*}
$$

on the closed left ideals $J$ of $A$ is a left complementor on $A$.

Lemma 4.2. Let $A$ be a complemented Banach*-algebra in which $x^{*} x=0$ implies $x=0$. Then every closed two-sided ideal I of $A$ is a complemented Banach*algebra.

Proof. Since $x^{*} x=0$ implies $x=0$, we have $r(A)=(0)$ and therefore, by [10] Lemma $1, l(I)=r(I)=I^{p}$ which also implies that $I$ is a complemented algebra. Now let $x \in I$ and $y \in I^{p}$. Then

$$
\left(x^{*} y\right)^{*}\left(x^{*} y\right)=y^{*} x x^{*} y \in I \cap I^{p}=(0),
$$

so that $\left(x^{*} y\right)^{*}\left(x^{*} y\right)=0$. Thus $x^{*} y=0$ and hence $x^{*} \in l\left(I^{p}\right)=I^{p p}=I$, for all $x \in I$. Therefore $I^{*}=I$.

THEOREM 4.3 (Structure Theorem). Let $A$ be a semi-simple right complemented Banach*-algebra in which $x^{*} x=0$ implies $x=0$. Then $A$ is the topological direct sum of its minimal closed two-sided ideals, each of which is a simple right complemented Banach*-algebra. 
Proof. Follows from Lemma 4.2 and [10] Theorem 4.

LEMMA 4.4. Let $A$ be a simple complemented Banach*-algebra in which $x^{*} x=0$ implies $x=0$. Then there exists a faithful ${ }^{*}$-representation of $A$ on a Hilbert space $H$ such that the image of $A^{\prime}$ of $A$ in $L(H)$ is a dense subalgebra of $L C(H) ; A$ is an $A^{*}$-algebra.

Proof. Let $I$ be a minimal left ideal of $A$. Since $I=A e$, where $e$ is a self-adjoint minimal idempotent, the scalar-valued function $(x, y)$ on $I$ given by $(x, y) e=$ $y^{*} x, x, y \in I$, is an inner product on $I$. Let $H$ be the completion of $I$ in the norm $|x|_{0}=(x, x)^{\frac{1}{2}}$. The left regular representation $x \rightarrow T_{x}$ of $A$ on $I$ is faithful and is a *-representation with respect to this inner product and, for each $x \in A, T_{x}$ is a bounded operator relative to the norm $|\cdot|_{0}$. Therefore $A$ has a faithful *-representation on $H$ whose image $A^{\prime}$ contains all operators of the form $g \otimes h, g, h \in I$ ([8] theorem (4.10.5)). Since $I$ is dense in $H, \operatorname{cl}\left(A^{\prime}\right) \supset L C(H)$. Now the socle $\subseteq$ of $A$ is dense in $A$ and every element of $\subseteq$ gives rise to an operator of finite rank on $I$ ([2] Lemma 5) and hence on $H$. Therefore $A^{\prime} \subset L C(H)$ and so $\operatorname{cl}\left(A^{\prime}\right)=L C(H)$.

THEOREM 4.5. Let A be a semi-simple complemented Banach*-algebra in which $x^{*} x=0$ implies $x=0$. Then $A$ is an $A^{*}$-algebra which is a dense subalgebra of a dual $B^{*}$-algebra $\mathfrak{A} ; A$ is uniquely determined up to ${ }^{*}$-isomorphism.

Proof. Let $\left\{I_{\lambda}: \lambda \in \Lambda\right\}$ be the family of all minimal closed two-sided ideals of $A$. By Lemma 4.4, each $I_{\lambda}$ may be identified with a dense subalgebra of $L C\left(H_{\lambda}\right)$, for some Hilbert space $H_{\lambda}$. Let $\mathfrak{A}=\left(\sum L C\left(H_{\lambda}\right)\right)_{0}$. By Theorem 4.3, $A$ can be identified as a subalgebra of $\mathfrak{A}$ so that $A$ is an $A^{*}$-algebra. Considering $A$ as a subalgebra of $\mathfrak{A}$, we have $L C\left(H_{\lambda}\right) \subset \operatorname{cl}(A)$ for all $\lambda$, and so $\mathfrak{A} \subset \operatorname{cl}(A)$, i.e., $A$ is dense in $\mathfrak{A}$. Since the socle is dense in $A$, by [6] Theorem $3, \mathfrak{A}$ is uniquely determined up to ${ }^{*}$-isomorphism.

THEOREM 4.6. Let $A$ be a complemented Banach*-algebra in which $x^{*} x=0$ implies $x=0$. Then the radical $\mathscr{R}$ and the ${ }^{*}$-radical $\mathscr{R}^{(*)}([8, \mathrm{p} .210])$ of $A$ coincide.

Proof. By [8] Theorem (4.4.10), $\mathscr{R}^{(*)} \supset \mathscr{R}$. We may assume $\mathscr{R} \neq A$; for if $\mathscr{R}=A$, then $\mathscr{R}=\mathscr{R}^{(*)}=A$. By $\left[10\right.$, Theorem 2] and Lemma $4.2, \mathscr{R}^{p}$ is a semisimple right complemented Banach*-algebra; clearly, $x^{*} x=0$ implies $x=0$ for all $x \in \mathscr{R}^{p}$. Hence, by Theorem $4.5, \mathscr{R}^{p}$ is an $A^{*}$-algebra. It is easy to show that the natural homomorphism $x \rightarrow x^{\prime}$ (where $x^{\prime}=x+\mathscr{R}$ ) is a ${ }^{*}$-isomorphism of $\mathscr{R}^{p}$ onto $A / \mathscr{R}$. Therefore $A / \mathscr{R}$ is an $A^{*}$-algebra and, by [8] Corollary (4.8.12), $A / \mathscr{R}$ is *-semi-simple. Hence $\mathscr{R}^{(*)} / \mathscr{R}=(0)$ and so $\mathscr{R}^{(*)}=\mathscr{R}$.

\section{Annihilator and weak $\left(\beta_{k}\right)$ properties in Banach*-algebras}

If $A$ is a Banach*-algebra in which $x^{*} x=0$ implies $x=0$, then, by [8] Lemma (4.10.1), every minimal left ideal $I$ of $A$ is of the form $I=A e$, where $e$ 
is a minimal self-adjoint idempotent. A similar result holds for minimal right ideals. It follows from the proof of [8] Theorem (4.10.3) that the scalar-valued function $(x, y)$ defined by $(x, y) e=y^{*} x(x, y \in I)$ is an inner product on $I$. Hence $|x|_{0}=$ $(x, x)^{\frac{1}{2}}$ is a norm on $I$. Since this inner product will be used on several occasions in the rest of the paper, to avoid repeating ourselves in the future we will adopt the following notation: the bracket $(\cdot)$ will always denote the inner product on the minimal left ideal $I$ defined by $(x, y) e=y^{*} x(x, y \in I)$ and $|\cdot|_{0}$ the inner product norm on $I$ given by $|x|_{0}=(x, x)^{\frac{1}{2}}$, for all $x \in I$.

It is easy to see that if $A$ is a $B^{*}$-algebra, then the norm $|\cdot|_{0}$ coincides with the given norm on every minimal left ideal of $A$.

Definition. A Banach*-algebra $A$ is said to have the weak $\left(\beta_{k}\right)$ property if, for every minimal left ideal $I$ of $A$, there exists a constant $k$ (depending on $I$ ) such that $\|x\|^{2} \leqq k\left\|x^{*} x\right\|$ for all $x \in I$.

ReMark. $A$ has the weak $\left(\beta_{k}\right)$ property if and only if every minimal left ideal $I$ is complete under the inner product norm $|\cdot|_{0}$, or equivalently, the norms $|\cdot|_{0}$ and $\|\cdot\|$ are equivalent on every minimal left ideal $I$ (see [8] Theorem (4.10.6) and its proof).

THEOREM 5.1. Let $A$ be an $A^{*}$-algebra which is a dense subalgebra of a $B^{*}$ algebra $\mathfrak{A}$. Then $A$ has the weak $\left(\beta_{k}\right)$ property if and only if every minimal left (right) ideal of $A$ is also a minimal left (right) ideal of $\mathfrak{A}$.

Proof. Suppose that every minimal left ideal of $A$ is also a minimal left ideal of $\mathfrak{A}$, and let $I$ be a minimal left ideal of $A$. Then $I$ is complete in the inner product norm $|\cdot|_{0}$. Hence by the above Remark, $A$ has the weak $\left(\beta_{k}\right)$ property. Conversely suppose $A$ has the weak $\left(\beta_{k}\right)$ property and let $I$ be a minimal left ideal of $A$. Then $I=A e$ with $e$ a self-adjoint idempotent in $A$. Since $e A e$ is one-dimensional and dense in $e \mathfrak{A} e, e$ is a minimal idempotent of $\mathfrak{A}$. But $|\cdot|_{0}$ and $|\cdot|$ are equal on $\mathfrak{A} e$ and $I$ is complete under $|\cdot|_{0}$. Since $I$ is dense in $\mathfrak{A} e$, we have $A e=\mathfrak{A} e$. The same argument holds for minimal right ideals.

COROLlaRY 5.2. Let $A$ be an $A^{*}$-algebra which is a dense two-sided ideal of a dual $B^{*}$-algebra $\mathfrak{X}$. Then $A$ has the weak $\left(\beta_{k}\right)$ property.

Proor. This follows from Theorem 5.1, since in this case $A$ and $\mathfrak{A}$ have the same minimal left (right) ideals.

Lemma 5.3. Let $A$ be a Banach*-algebra with socle $\subseteq$ such that $a \subseteq=(0)$ implies $a=0$. If $A$ has the weak $\left(\beta_{k}\right)$ property, then $x^{*} x=0$ implies $x=0$.

ProOF. By [8] Corollary (2.5.8), $A$ has a unique norm topology and hence the involution is continuous. Let $x \in A$ be such that $x^{*} x=0$, and let $I$ be any minimal left ideal of $A$. Then, for each $a \in I,(x a)^{*}(x a)=a^{*} x^{*} x a=0$. Hence by the weak $\left(\beta_{k}\right)$ property of $A,\|x a\|^{2}=0$ which gives $x a=0$ and therefore $x I=(0)$. As $I$ 
is an arbitrary minimal left ideal of $A$, it follows that $x \widetilde{S}=(0)$ and consequently $x=0$.

THeOREM 5.4. Let $A$ be a semi-simple Banach*-algebra. Then the following statements are equivalent:

(i) $A$ is an annihilator algebra in which $x^{*} x=0$ implies $x=0$.

(ii) $A$ has the waak $\left(\beta_{k}\right)$ property and the socle $\subseteq$ of $A$ is dense in $A$.

Proof. (i) $\Rightarrow$ (ii). Suppose (i) holds. By [2] Theorem 4, the socle $\subseteq$ of $A$ is dense in $A$ and therefore the involution is continuous. Let $I$ be a minimal left ideal of $A ; I=A e$, where $e$ is a self-adjoint idempotent. Let $J$ be the closed twosided ideal generated by $I$. Then $J$ is a minimal closed two-sided ideal of $A$ ([2] Theorem 5) with $J^{*}=J$ and therefore a simple annihilator Banach*-algebra; moreover, $I$ is a minimal left ideal of $J$. Applying the proof of [8] Theorem (4.10.16) to $J$ and $I$, we see that $I$ is complete under the inner product norm $\mid \cdot l_{0}$ and so $A$ has the weak $\left(\beta_{k}\right)$ property.

(ii) $\Rightarrow$ (i). Suppose (ii) holds. By Lemma 5.3, $x^{*} x=0$ implies $x=0$. Assume first that $A$ is simple, and let $I$ be a minimal left ideal of $A$. Since $A$ has the weak $\left(\beta_{k}\right)$ property, $I$ is a Hilbert space under the inner product $(\cdot)$. Therefore the image $A^{\prime}$ of $A$ by the left regular representation $x \rightarrow T_{x}$ of $A$ on $I$ contains the set $F$ of all operators of finite rank on $I$ (see the proof of Lemma 4.4). But the elements of the socle give rise to operators of finite rank on $I$ and, since $A=\operatorname{cl}(\subseteq), F$ is dense in $A^{\prime}$ relative to the norm $\|\cdot\|$. Hence, by [8] Theorem (2.8.23), $A^{\prime}$ is an annihilator algebra and therefore $A$ is an annihilator algebra, since the representation is faithful.

Now suppose that $A$ is not simple. Let $I$ be a minimal left ideal of $A$ and $J$ the closed two-sided ideal generated by $I$. Then $J$ is a minimal closed two-sided ideal of $A$ (see the proof of Lemma 2.1) with $J^{*}=J$. Since $A=\operatorname{cl}(\subseteq)$, Lemma 2.1 shows that $I$ is a minimal left ideal of $J$ and since $J$ is simple, $J$ has a dense socle and so is an annihilator algebra by the argument above. Thus, by [8] Theorem (2.8.29), $A$ is an annihilator algebra.

Lemma 5.5. Let $A$ be an annihilator $A^{*}$-algebra, $I$ a closed right ideal of $A$ and $\mathfrak{A}$ the completion of $A$ in an auxiliary norm $|\cdot|$. Then the following statements are true:

(i) $\mathfrak{A}$ is a dual $B^{*}$-algebra which is uniquely determined up to *-isomorphism.

(ii) $A$ and $\mathfrak{A}$ have the same socle.

(iii) If $\subseteq$ is the socle of $A$, then $\operatorname{cl}(I) \subseteq \subset I$.

(iv) $l(\mathrm{cl}(I))=\operatorname{cl}\left(l_{A}(I)\right)$.

(v) $\operatorname{cl}(I) \cap A=r_{A}\left(l_{A}(I)\right)$.

$\left(\right.$ Where $\operatorname{cl}(S)\left(\right.$ resp. $\left.\mathrm{cl}_{A}(S)\right)$ denotes the closure of the set $S$ in $\mathfrak{A}($ resp. $A)$ and $l(S)$ (resp. $\left.l_{A}(S)\right)$ the left annihilator of $S$ in $\mathfrak{A}($ resp. $A)$.) 
Proof. (i). Since $\mathbb{S}$ is dense in $A, A$ has a unique auxiliary norm and therefore $\mathfrak{A}$ is uniquely determined up to *-isomorphism (see the proof of Theorem 4.5). Since $A$ has the weak $\left(\beta_{k}\right)$ property, $\subseteq$ is contained in the socle of $\mathfrak{A}$ by Theorem 5.1. Thus the socle of $\mathfrak{A}$ is dense in $\mathfrak{A}$ and so $\mathfrak{A}$ is dual by [5] Theorem 2.1.

(ii) By the weak $\left(\beta_{k}\right)$ property, $\widetilde{S}$ is a two-sided ideal of $\mathfrak{A}$. Let $f$ be a minimal idempotent in $\mathfrak{A}$. Then clearly $I=f \mathfrak{A} \cap S$ is a non-zero right ideal of $\mathfrak{A}$ contained in $A$. As $f \mathfrak{A}$ is a minimal right ideal of $\mathfrak{A}, f \mathfrak{A}=I \subset A$ and so $f \in A$. This proves (ii).

(iii). It clearly suffices to show that $x y e \in I$ for $x \in \operatorname{cl}(I), y \in A$ and $e$ a minimal idempotent. Now $A e=\mathfrak{A} e$ and the two norms $|\cdot|$ and $\|\cdot\|$ are equivalent on $A e$ (by the weak $\left(\beta_{k}\right)$ property in $A$ ). Hence

$$
\|x y e\| \leqq c|x|\|y e\|,
$$

for some constant $c$. Let $\left\{x_{n}\right\}$ be a sequence in $I$ such that $\left|x_{n}-x\right| \rightarrow 0$ as $n \rightarrow \infty$. Since

$$
\left\|x_{n} y e-x y e\right\| \leqq c\left|x_{n}-x\right|\|y e\|,
$$

$\left\|x_{n} y e-x y e\right\| \rightarrow 0$ as $n \rightarrow \infty$, which shows that $x y e \in I$. Hence $\operatorname{cl}(I) \subseteq \subset I$.

(iv). Let $\left\{e_{\beta}\right\}$ be the set of all minimal idempotents in $l(\mathrm{cl}(I))$;

$$
e_{\beta} \in l(\mathrm{cl}(I)) \cap A=l(I) \cap A=l_{A}(I),
$$

for all $\beta$. Now $\operatorname{cl}\left(\sum_{\beta} \mathfrak{A} e_{\beta}\right)=l(\operatorname{cl}(I))$ (Lemma 3.1) and so

$$
\left.\left.\operatorname{cl}\left(l_{A}\right) I\right)\right) \supset \operatorname{cl}\left(\sum_{\beta} A e_{\beta}\right)=\operatorname{cl}\left(\sum_{\beta} \mathfrak{A} e_{\beta}\right)=l(\operatorname{cl}(I)) .
$$

But $l_{A}(I) \subset l(\operatorname{cl}(I))$. Hence $\operatorname{cl}\left(l_{A}(I)\right)=l(\operatorname{cl}(I))$.

(v) By the duality of $\mathfrak{A}$ and (iv), we have

$$
r_{A}\left(l_{A}(I)\right)=r\left(l_{A}(I)\right) \cap A=r(l(\mathrm{cl}(I))) \cap A=\mathrm{cl}(I) \cap A .
$$

This completes the proof.

From Theorem 4.5 and Lemma 5.5 we see that if $A$ is either a complemented or an annihilator $A^{*}$-algebra, then $A$ can be imbedded as a dense subalgebra in a unique (up to ${ }^{*}$-isomorphism) $B^{*}$-algebra $\mathfrak{A}$. From now on we shall refer to $\mathfrak{A}$ as the completion of $A$.

THEOREM 5.6. Let $A$ be a semi-simple complemented Banach*-algebra with the weak $\left(\beta_{k}\right)$ property. Then $A$ is a dual $A^{*}$-algebra.

Proof. We use the notation of Lemma 5.5. Since the socle is dense, Theorems 4.5 and 5.4 show that $A$ is an annihilator $A^{*}$-algebra. Let $\mathfrak{A}$ be the completion of $A$ and let $I$ be a closed right ideal of $A$. We claim that $\operatorname{cl}(I) \cap A=I$. Let $J=\operatorname{cl}(I) \cap A$. Then $J$ is a closed right ideal of $A$, and clearly $I \subset J$. Let $p$ ba the given complementor on $A$ and let $\left\{e_{\alpha}\right\}$ be the family of all minimal $p$-projections 
in $I$. If $I \neq J$, then $I^{p} \cap J \neq(0)$ and so, by Lemma 3.1, contains a minimal $p$-projection $f$. Since $e_{\alpha} \in I$ and $f \in I^{p}$, we have $e_{\alpha} f=f e_{\alpha}=0$ for all $\alpha$ and hence, since $\operatorname{cl}(I)=\operatorname{cl}\left(\sum_{\alpha} e_{\alpha} A\right)$, it follows that $f \operatorname{cl}(I)=(0)$. But this is a contradiction since $f \in \operatorname{cl}(I)$ and $f^{2}=f \neq 0$. Hence $J=I$ and consequently, by Lemma 5.5, $I=l_{A}\left(r_{A}(I)\right)$. Applying now the continuity of the involution, we obtain that $A$ is dual.

COROLlary 5.7. An annihilator complemented $A^{*}$-algebra is dual.

Proof. Follows from Theorems 5.4 and 5.6.

From Theorem 3.3 and Corollary 5.7, we have the following result:

THEOREM 5.8. Let $A$ be a complemented $A^{*}$-algebra with a complementor $p$. Then the following statements are equivalent:

(i) $A$ is dual.

(ii) Every non-zero right ideal contains a minimal p-projection.

(iii) Every maximal closed right ideal is modular.

(iv) Every maximal closed right ideal has non-zero left annihilator.

Definition. A Banach algebra $A$ is said to be completely continuous (c.c.) if the left- and right-multiplication operators of every element in $A$ are completely continuous on $A$.

THEOREM 5.9. A complemented c.c. $A^{*}$-algebra is dual.

Proof. By Theorem 4.3, $A$ is the direct topological sum of all its minimal closed two-sided ideals $I_{\lambda}$, each of which is a simple c.c. complemented $A^{*}$-algebra. Since each $I_{\lambda}$ is finite dimensional, it is a full matrix algebra and hence dual. Therefcre, by [8] Theorem (2.8.9), $A$ is an annihilator algebra and so, by Corollary $5.7, A$ is dual.

\section{Weakly completely continuous $\boldsymbol{A}^{*}$-algebras}

Definition. A Banach algebra is said to be weakly completely continuous (w.c.c.) if the left- and right-multiplication operators of every element in $A$ are weakly completely continuous on $A$.

THEOREM 6.1. An annihilator $A^{*}$-algebra $A$ is w.c.c.

Proof. Let $\mathfrak{A}$ be the completion of $A$. $\mathfrak{U}$ is dual and hence w.c.c. by [6] Theorem 6. Let $e$ be a minimal idempotent of $A$. From Lemma 5.5 we have $e A=$ $\mathfrak{A} e$ and from its proof that $\|e x\| \leqq c|| e \||x|$ for all $x \in \mathfrak{A}$ (see the proof of (iii)). Let $y \in A$ and let $\left\{y_{n}\right\}$ be any bounded sequence in $A$. As $\mathfrak{A}$ is w.c.c. and $\left\{y_{n}\right\}$ is bounded in $|\cdot|$, there exists a subsequence $\left\{y_{n_{k}}\right\}$ such that $\left\{y y_{n_{k}}\right\}$ converges weakly 
to an element $z \in \mathfrak{A}$. For each continuous linear functional $f$ on $A$ let $g$ be the linear functional on $\mathfrak{A}$ given by $g(x)=f(e x)(x \in \mathfrak{A})$. Since

$$
|g(x)|=|f(e x)| \leqq\|f\|\|e x\| \leqq c\|f\|\|e\||x|(x \in \mathfrak{U}),
$$

where $\|f\|$ denotes the norm $\mathrm{cf} f$ with respect to $\|\cdot\|$, it follows that $g$ is continuous on $\mathfrak{A}$. Now $e z \in A$ and

$$
f\left(e y y_{n_{k}}-e z\right)=g\left(y y_{n_{k}}-z\right) \rightarrow 0 \text { as } n \rightarrow \infty,
$$

and so $e y$ is a w.c.c. element of $A$. This shows that every element of the socle $\subseteq$ of $A$ is w.c.c. Since $\mathbb{S}$ is dense in $A$ and the set of all w.c.c. elements is closed in $A, A$ is w.c.c.

THEOREM 6.2. Let $A$ be an $A^{*}$-algebra which is a dense two-sided ideal of a $B^{*}$-algebra $\mathfrak{A}$. Then $A$ is an annihilator algebra if and only $A$ is w.c.c. and $A^{2}$ is dense in $A$.

Proof. If $A$ is an annihilator algebra, Theorem 6.1 shows that $A$ is w.c.c., and since $A^{2}$ contains the socle of $A, A^{2}$ is dense in $A$. Conversely, suppose that $A$ is w.c.c. and $A^{2}$ is dense in $A$. Then, by [6] Lemma $9, \mathfrak{A}$ is w.c.c. (therefore dual) and hence, by Corollary 5.2, $A$ has the weak $\left(\beta_{k}\right)$ property. Let $\subseteq$ be the socle of $A$ and let $\left\{e_{\alpha}\right\}$ be a maximal orthogonal family of minimal self-adjoint idempotents in $A$. Then, for all $x, y \in A$, we have $x y=\sum e_{x} x y$, the summation being taken relative to the norm $\|\cdot\|$ (see the proof of [6] Theorem 16). Thus (in the notation of Lemma 5.5) we have that $x y \in \mathrm{cl}_{A}(\widetilde{S})$, which shows that $\mathrm{cl}_{A}(\widetilde{S})=\mathrm{cl}_{A}\left(A^{2}\right)=A$. Theorem 5.4 now completes the proof.

\section{Dual $\boldsymbol{A}^{*}$-algebras}

In this section we shall give several characterizations of duality in $A^{*}$-algebras.

THEOREM 7.1. Let $A$ be an annihilator $A^{*}$-algebra. Then the following statements are equivalent:

(i) $A$ is dual.

(ii) $x$ belongs to the closure of $x A$ for every $x$ in $A$.

(iii) For every closed right ideal I of $A$ and $x \in A, x x^{*} \in I$ implies $x \in I$.

(iv) Every closed right ideal $I$ of $A$ is the intersection of maximal closed right ideals containing it.

Proof. We use the notation of Lemma 5.5. Let $\mathfrak{A}$ be the completion of $A$ and $\mathfrak{S}$ the socle of $A ; \mathfrak{A}$ is dual and $\mathrm{cl}_{A}(x \subseteq)=\operatorname{cl}_{A}(x A)$ for all $x \in A$. In the ensuing arguments let $I$ be a closed right ideal of $A$ and $R=\operatorname{cl}(I)$.

(i) $\Rightarrow$ (ii). This is [8] Corollary (2.8.3). 
(ii) $\Rightarrow$ (iii). Suppose $x x^{*} \in I$. Then $x x^{*} \in R$ and therefore, since $R$ is a closed right ideal of $\mathfrak{A}$, [8] Corollary (4.9.3) implies that $x \in R \cap A$. Hence, if $x \in \mathrm{cl}_{A}(x A)$, then $x \in \mathrm{cl}_{A}(R \subseteq) \subset I$ by Lemma 5.5 (ii), whence (iii).

(iii) $\Rightarrow$ (iv). Suppose (iii) holds and let $x \in R \cap A$. Then clearly $x \in \mathrm{cl}_{A}(x A)$ and so $x \in I$ by the argument above. Hence $I=R \cap A$. Now, by [3] Theorem (2.9.5) (iii), $R=\bigcap_{\alpha} \mathfrak{M}_{\alpha}$, where $\left\{\mathfrak{M}_{\alpha}\right\}$ is the family of all maximal closed right ideals of $A$ containing $R$. Therefore $I=\bigcap_{\alpha}\left(\mathfrak{M}_{\alpha} \cap A\right)$. [2], Theorem 1 and Lemma 5.5 (ii) show that each $M_{\alpha}=\mathfrak{M}_{\alpha} \cap A$ is a maximal closed right ideal of $A$, whence (iv).

(iv) $\Rightarrow$ (i). Suppose (iv) holds. Since every maximal closed right ideal $M$ of $A$ is of the form $M=\{x-e x: x \in A\}$, where $e$ is a minimal idempotent, $\operatorname{cl}(M)$ is a maximal closed right ideal of $\mathfrak{A}$ and clearly $\operatorname{cl}(M) \cap A=M$. Hence if $\left\{M_{\alpha}\right\}$ is the family of all closed right ideals of $A$ containing $I$ and $\mathfrak{M}_{\alpha}=\operatorname{cl}\left(M_{\alpha}\right)$ for each $\alpha$, then $R=\bigcap_{\alpha} \mathfrak{M}_{\alpha}$ and $R \cap A=\bigcap_{\alpha}\left(\mathfrak{M}_{\alpha} \cap A\right)=\bigcap_{\alpha} M_{\alpha}=I$. Therefore, by Lemma $5.5(\mathrm{v})$ and the continuity of the involution, $A$ is dual.

THEOREM 7.2. Let $A$ be an $A^{*}$-algebra which is a dense two-sided ideal of a $B^{*}$-algebra $\mathfrak{Y}$. Then $A$ is dual if and only if every maximal commutative *-subalgebra of $A$ is dual.

Proof. If $A$ is dual then, by [6] Theorem 19, every maximal commutative *-subalgebra of $A$ is also dual. Conversely suppose that every maximal commutative *-subalgebra of $A$ is dual. Let $\subseteq$ be the socle of $A$ (and hence of $\mathfrak{A}$ ). Let $x \in A$ and write $x=x_{1}+i x_{2}$, where $x_{1}$ and $x_{2}$ are hermitian elements of $A$ and let $B_{1}$ and $B_{2}$ be maximal commutative ${ }^{*}$-subalgebras containing $x_{1}, x_{2}$ respectively. Since $B_{1}, B_{2}$ have dense socles, it follows that $x_{1}$ and $x_{2}$ belong to $\mathrm{cl}_{A}(\Im)$. Hence $x \in \mathrm{cl}_{A}(\Im)$ and so $\mathrm{cl}_{A}(\widetilde{S})=A$. It follows now that $\subseteq$ is dense in $\mathfrak{A}$ and consequently $\mathfrak{A}$ is dual by [5] Theorem 2.1. Therefore by Corollary 5.2 and Theorem 5.4, $A$ is an annihilator algebra. Since $B_{i}$ is dual, $x_{i} \in \operatorname{cl}_{A}\left(x_{i} B_{i}\right) \subset \operatorname{cl}_{A}\left(x_{i} A\right)(i=1,2)$. Let $\left\{e_{\alpha}\right\}$ be a maximal orthogonal family of minimal self-adjoint idempotents in $A$. By the proof of [6] Theorem 16, $x_{i}=\sum_{\alpha} e_{\alpha} x_{i}(i=1,2)$ in the norm $\|\cdot\|$ and hence $x=\sum_{\alpha} e_{\alpha} x$ in the norm $\|\cdot\|$. Therefore $x \in \mathrm{cl}_{A}(x A)$ and so, by Theorem 7.1, $A$ is dual. This completes the proof.

THEOREM 7.3. Let $A$ be an $A^{*}$-algebra which is a dense two-sided ideal of a $B^{*}$-algebra $\mathfrak{A}$. Then $A$ is dual if and only if it is complemented.

Proof. We use the notation of Lemma 5.5. Suppose $A$ is complemented. By Theorem $4.5, \mathfrak{A}$ is dual and therefore, by Corollary $5.2, A$ has the weak $\left(\beta_{k}\right)$ property. Theorem 5.6 now shows that $A$ is dual. Conversely, suppose $A$ is dual. Let $I$ be a closed right ideal of $A$ and let $R=\operatorname{cl}(I) ; R$ is a closed right ideal of $\mathfrak{A}$. Let $\left\{e_{\alpha}\right\}$ be a maximal orthogonal family of minimal self-adjoint idempotents contained in $R$. By Lemma 5.5, $\left\{e_{\alpha}\right\} \subset R \cap A=I$. Now $\mathfrak{A}=R+l(R)^{*}$, so that 
$x=y+z$ with $y \in R$ and $z \in l(R)^{*}$, for every $x \in A$. Hence $e_{\alpha} x=e_{\alpha} y$ for all $e_{\alpha}$ and so, by [6] Lemma 6,y $=\sum e_{\alpha} y=\sum e_{\alpha} x$, where the summations are taken in the norm $|\cdot|$. Since, by [6] Theorem $16, \sum e_{x} x$ is also summable in $\|\cdot\|, y \in A \cap R=I$. Hence $z \in A \cap l(R)^{*}=l_{A}(I)^{*}$. Thus $A=I+l_{A}(I)^{*}$. It is easy to see that the mapping $I \rightarrow l_{A}(I)^{*}$ also has properties $\left(C_{1}\right),\left(C_{3}\right)$ and $\left(C_{4}\right)$. Hence $A$ is complemented.

We shall need the following result in 8 .

THEOREM 7.4. Every complemented $A^{*}$-algebra $A$ which is a dense two-sided ideal of $L C(H)$ is a two-sided ideal of $L(H)$.

Proof. By Theorem 7.3, $A$ is dual. Let $x \in A, y \in L(H)$ and let $\left\{e_{\alpha}\right\}$ be a maximal orthogonal family of minimal selfadjoint idempotents in $A$. By [6] Theorem $16, \sum_{\alpha} e_{\alpha} x$ is summable to $x$ in the norm $\|\cdot\|$ and hence there is only a countable number of $e_{\alpha}$ for which $e_{\alpha} x \neq 0$, say $e_{\alpha_{1}}, e_{\alpha_{2}}, \cdots$. Clearly $y e_{\alpha_{i}} \in A$ $(i=1,2, \cdots)$. For any two positive integers $m, n(m \leqq n)$, [6] Lemma 4 shows that

$$
\begin{aligned}
& \left\|\sum_{i=1}^{n} y e_{\alpha_{i}} x-\sum_{i=1}^{m} y e_{\alpha_{i}} x\right\|=\left\|\left(y \sum_{i=m+1}^{n} e_{\alpha_{i}}\right)\left(\sum_{i=m+1}^{n} e_{\alpha_{i}} x\right)\right\| \\
& \leqq k\left|y \sum_{i=m+1}^{n} e_{\alpha_{i}}\right|\left\|\sum_{i=m+1}^{n} e_{\alpha_{i}} x\right\| \leqq k|y|\left\|\sum_{i=m+1}^{n} e_{\alpha_{i}} x\right\|,
\end{aligned}
$$

where $k$ is a constant. Therefore $\left\{\sum_{i=1}^{n} y e_{\alpha_{i}} x\right\}$ is a Cauchy sequence in $A$ and so there exists an element, $z \in A$ such that $z=\sum_{i=1}^{\infty} y e_{\alpha_{i}} x$. Since $\sum_{i=1}^{\infty} e_{\alpha_{i}} x$ also converges to $x$ in the norm $|\cdot|$, we have $y x=\sum_{i=1}^{\infty} y e_{\alpha_{i}} x$. Hence $y x=z \in A$. Similarly we can show that $x y \in A$, and this completes the proof.

\section{Induced complementors}

Throughout this section we shall use the notation introduced in Lemma 5.5.

Let $A$ be an $A^{*}$-algebra which is a dense subalgebra of a $B^{*}$-algebra $\mathfrak{A}$. Let $p$ be a complementor on $\mathfrak{A}$ and $q$ a complementor on $A$. In this section we are going to give conditions on $A, \mathfrak{A}$ and the complementors $p$ and $q$ such that: (a) The mapping $q: I \rightarrow \operatorname{cl}(I)^{p} \cap A$ on the closed right ideals $I$ of $A$ is a complementor on $A$. (b) The mapping $\boldsymbol{p}: R \rightarrow \operatorname{cl}\left((R \cap A)^{q}\right)$ on the closed right ideals $R$ of $\mathfrak{A}$ is a complementor on $\mathfrak{A}$.

We shall say that the complementor $q$ is induced on $A$ by $p$ and the complementor $\boldsymbol{p}$ is induced on $\mathfrak{A}$ by $q$.

Lemma 8.1. Let $A$ be a dual $A^{*}$-algebra which is a dense two-sided ideal of the $B^{*}$-algebra $L C(H)$. Then, for every complementor $p$ on $L C(H)$, the mapping $q: I \rightarrow \operatorname{cl}(I)^{p} \cap A$ on the closed right ideals $I$ of $A$ is a complementor on $A$.

Proof. Let $p$ be a complementor on $L C(H)$. If the dimension of $H$ is finite, 
then $A=L C(H)$ and therefore $q=p$, so that $q$ is a complementor on $A$. Now suppose the dimension of $H$ is infinite. Then, by [1] Theorem $6.8, p$ is continuous and hence, by [1] Theorem 6.11, there exists an involution ${ }^{* \prime}$ on $L C(H)$ such that $R^{p}=l(R)^{* \prime}$, for every closed right ideal $R$ of $L C(H)$. This means, by [1] Corollary 6.14 , that there exists a positive operator $Q \in L(H)$ with continuous inverse $Q^{-1}$ such that $a^{* \prime}=Q^{-1} a^{*} Q$ for all $a \in L C(H)$. Now, from Theorem 7.4 we know that $A$ is a two-sided ideal of $L(H)$. Hence $a^{* \prime} \in A$ fcr all $a \in A$ and therefore $A$ is an $A^{*}$-algebra under the involution ${ }^{* \prime}$ (and an auxiliary norm $|\cdot|^{\prime}$ equivalent to $\left.|\cdot|\right)$. Since $A$ is dual, $I \rightarrow l_{A}(I)^{* \prime}$ is a complementor on $A$ (see the proof of Theorem 7.3) and we have

$$
\begin{aligned}
I^{q}=\operatorname{cl}(I)^{p} \cap A=l(\mathrm{cl}(I))^{* \prime} \cap A & =(l(\operatorname{cl}(I)) \cap A)^{* \prime} \\
& =l_{A}(I)^{* \prime} .
\end{aligned}
$$

Thus $q$ is a complementor on $A$ and the proof is complete.

Definition. Let $p$ be a complementor on a $B^{*}$-algebra $A$ and $P$ the $p$-derived mapping (see [1] Definition 3.7). We shall say that $p$ is uniformly continuous if $P$ is uniformly continuous.

THEOREM 8.2. Let $A$ be a dual $A^{*}$-algebra which is a dense two-sided ideal of a $B^{*}$-algebra $\mathfrak{A}$. Suppose that $\mathfrak{A}$ has no minimal left ideals of dimension less than three. Then, for every uniformly continuous complementor $p$ on $\mathfrak{A}$, the mapping $q$ : $I \rightarrow \mathrm{cl}(I)^{p} \cap A$ on the closed right ideals $I$ of $A$ is a complementor on $A$.

Proof. Let $p$ be a uniformly continuous complementor on $\mathfrak{A}$. Let $\left\{I_{\lambda}: \lambda \in \Lambda\right\}$ be the family of all minimal closed two-sided ideals of $A$. It is easy to see that, for each $\lambda, \operatorname{cl}\left(I_{\lambda}\right)$ is a minimal closed two-sided ideal of $\mathfrak{A}$ and hence *-isomorphic to $L C\left(H_{\lambda}\right)$, for some Hilbert space $H_{\lambda}$. Since $A$ is the direct topological sum of $I_{\lambda}$, $\mathfrak{A}$ is *-isomorphic to $\left(\sum L C\left(H_{\lambda}\right)\right)_{0}$. In the rest of the proof we identify $\mathfrak{A}$ with $\left(\sum L C\left(H_{\lambda}\right)\right)_{0}$. For each $\lambda$, let $p_{\lambda}$ be the complementor on $L C\left(H_{\lambda}\right)$ induced by $p$. Then, by [1] Theorem 3.9, each $p_{\lambda}$ is continuous on $L C\left(H_{\lambda}\right)$. Therefore each $p_{\lambda}$ gives rise to an involution ${ }_{\lambda}^{* \prime}$ on $L C\left(H_{\lambda}\right)$ and a positive operator $Q_{\lambda} \in L\left(H_{\lambda}\right)$ with continuous inverse $Q_{\lambda}^{-1}$ such that

$$
a_{\lambda}^{* \prime \lambda}=Q_{\lambda}^{-1} a_{\lambda}^{*} Q_{\lambda}
$$

for all $a_{\lambda} \in L C\left(H_{\lambda}\right)$ (see the proof of Lemma 8.1); we may clearly take $\left|Q_{\lambda}\right|=1$, for all $\lambda$. By the proof of [1] Theorem 7.4, $a \rightarrow a^{*^{\prime}}=\left(a_{\lambda}^{* \lambda}\right)$ is an involution on $\mathfrak{A}$ under which $\mathfrak{Q}$ is a $B^{*}$-algebra and $R^{p}=l(R)^{* \prime}$, for all closed right ideals $R$ of $\mathfrak{A}$. We show that $A$ is closed under the involution *'. Let $H=\oplus_{\lambda} H_{\lambda}$, the Hilbert direct sum of $H_{\lambda}$ and $Q=\left(Q_{\lambda}\right)$. Then $Q$ is a positive operator in $L(H)$ with bounded inverse and $|Q|=1$. Let $\left\{e_{\alpha}\right\}$ be a maximal orthogonal family of minimal self-adjoint idempotents in $A$. Since $\sum_{\alpha} e_{\alpha} x$ converges to $x$ in the norm $\|\cdot\|$, $e_{\alpha} x \neq 0$ for only a countable number of $e_{\alpha}$, say $e_{x_{1}}, e_{\alpha_{2}}, \cdots$. Now each $e_{\alpha_{i}}$ belongs 
to some $I_{\lambda}$ and $Q I_{\lambda}=Q_{\lambda} I_{\lambda} \subset I_{\lambda}$ (by Theorem 7.4); hence each $Q e_{\alpha_{i}} \in A$ and so $\sum_{i=1}^{n} Q e_{\alpha_{i}} x \in A$ for $n=1,2, \cdots$ (we identify $A$ as a subalgebra of $L(H)$.) Since $\left\{\sum_{i=1}^{n} Q e_{\alpha_{i}} x\right\}$ converges to $Q x$ in the norm $\|\cdot\|$ (see the proof of Theorem 7.4), $Q x \in A$ and so $x^{*} Q=(Q x)^{*} \in A$; similarly $Q^{-1} x \in A$. Therefore $x^{* \prime}=$ $Q^{-1} x^{*} Q \in A$, for all $x \in A$. Thus ${ }^{* \prime}$ is an involution on $A$ and therefore, since $A$ is dual, $I \rightarrow l\left(I_{A}\right)^{* \prime}$ is a complementor on $A$. Now, applying the argument in the proof of Lemma 8.1, we obtain that $I^{q}=\operatorname{cl}(I)^{p} \cap A=l_{A}(I)^{* \prime}$, which shows that $\boldsymbol{q}$ is a complementor on $A$.

Corollary 8.3. Let $A, \mathfrak{A}, p$ and $q$ be as in Theorem 8.2. Then there exists an involution ${ }^{* \prime}$ in $A$ such that $I^{q}=l_{A}(I)^{* \prime}$ for every closed right ideal $I$ of $A$.

NotATion. Let $A$ be an algebra of operators on a normed space $X$. For every closed subspace $S$ of $X$, let

$$
\mathscr{J}_{A}(S)=\{a \in A=a(X) \subseteq S\} .
$$

For every right ideal $I$ of $A$, let $\mathscr{S}_{A}(I)$ be the smallest closed subspace of $X$ that contains the range $a(X)$ of each operator $a$ in $I$. We shall write $\mathscr{J}(S)$ for $\mathscr{J}_{A}(S)$ and $\mathscr{S}(I)$ for $\mathscr{S}_{A}(I)$ if $A=L C(H)$ and $X=H$.

LemMA 8.4. Let $A$ be a dual $A^{*}$-algebra which is a dense subalgebra of $L C(H)$. Then, for every closed right ideal $I$ of $A, I=\mathscr{J}_{A}\left(\mathscr{S}_{A}(I)\right)$ and, for every closed subspace $S$ of $H, \mathscr{J}_{A}(S)$ is a closed right ideal of $A$ and $S=\mathscr{S}_{A}\left(\mathscr{J}_{A}(S)\right)$.

Proof. It is easy to see that $A$ is simple and that the set of all operators of finite rank on $H$ is dense in $A$. The proof can now be completed by using the argument (with obvious modifications) given in the proof of [1] Lemma 4.1.

ReMARK. Lemma 8.4 shows that $I \rightarrow \mathscr{S}_{A}(I)$ defines a one-to-one correspondence between the closed right ideals of $A$ and the closed subspaces of $H$. Moreover if $q$ is a complementor on $A$, then the mapping

$$
S \rightarrow S^{\prime}=\mathscr{S}_{A}\left(\mathscr{J}_{A}(S)^{q}\right)
$$

defines a complementor on the closed subspaces $S$ of $H$ in the sense of [4] Theorem 1.

LemMA 8.5. Let $A$ be a dual $A^{*}$-algebra which is a dense subalgebra of $L C(H)$. Then, for every complementor $q$ on $A$, the mapping $p: R \rightarrow \operatorname{cl}\left((R \cap A)^{q}\right)$ on the closed right ideals $R$ of $L C(H)$ is a complementor on $L C(H)$.

Proof. It is clear that $A$ is simple. Let $q$ be a complementor on $A$. Then, by the Remark above, the mapping $S \rightarrow S^{\prime}=\mathscr{S}_{A}\left(\mathscr{J}_{A}(S)^{q}\right)$ defines a complementor on the closed subspaces $S$ of $H$. By the Remark following [1] Lemma 4.1, the mapping $S \rightarrow S^{\prime}$ induces a complementor $p^{\prime}$ on $L C(H)$ given by the relation $R^{p^{\prime}}=J\left(S(R)^{\prime}\right)$, for every closed right ideal $R$ of $L C(H)$. It is easy to see that 
$\operatorname{cl}(R \cap A)=R$. In fact, let $\mathfrak{A}=L C(H)$ and let $\left\{e_{\alpha}\right\}$ be the family of all minimal self-adjoint idempotents in $R$. Then clearly $R=\operatorname{cl}\left(\sum_{\alpha} e_{\alpha} \mathfrak{A}\right)$. But from Lemma 5.5 we have $e_{\alpha} \mathfrak{A} \subset R \cap A$ for all $\alpha$; hence $R=\operatorname{cl}(R \cap A)$. Similarly $R^{p^{\prime}}=\operatorname{cl}\left(R^{p^{\prime}} \cap A\right)$. Now $\mathscr{J}_{A}(S)=\mathscr{J}(S) \cap A$ and, by Lemma 8.4,

$$
\mathscr{J}_{A}(\mathscr{S}(R))=\mathscr{J}(\mathscr{S}(R)) \cap A=R \cap A=I .
$$

Therefore

$$
\begin{aligned}
R^{p^{\prime}} \cap A & =\mathscr{J}\left(\mathscr{S}(R)^{\prime}\right) \cap A=\mathscr{J}\left(\mathscr{S}_{A}\left(\mathscr{J}_{A}(\mathscr{S}(R))^{q}\right)\right) \cap A=\mathscr{J}\left(\mathscr{S}_{A}\left(I^{q}\right)\right) \cap A \\
& =\mathscr{J}_{A}\left(\mathscr{S}_{A}\left(I^{q}\right)\right)=I^{q}=(R \cap A)^{q} .
\end{aligned}
$$

Hence $R^{p^{\prime}}=\operatorname{cl}\left(R^{p^{\prime}} \cap A\right)=\operatorname{cl}\left((R \cap A)^{q}\right.$, so that $p$ is a complementor on $\mathfrak{A}$.

LeMma 8.6. Let $\mathfrak{A}$ be a $B^{*}$-algebra which has no minimal left ideals of dimension less than three. Let $p$ be a continuous complementor on $\mathfrak{A}$ and let $\mathscr{E}_{p}$ be the set of all minimal p-projections in $\mathfrak{A}$. Then $p$ is uniformly continuous if and only if the set $\left\{|e|: e \in \mathscr{E}_{p}\right\}$ is bounded.

Proof. Suppose $p$ is uniformly continuous. By [1] Theorem 7.4, there exists an involution ${ }^{* \prime}$ on $\mathfrak{A}$ for which $R^{p}=l(R)^{* \prime}$, for every closed right ideal $R$ of $\mathfrak{A}$, and an equivalent norm $|\cdot|$ ' on $\mathfrak{A}$ satisfying the $B^{*}$-condition for *'. Since, by [1] Corollary 4.4, $e^{* \prime}=e$ and hence $|e|^{\prime}=1$, it follows that $\left\{|e|: e \in \mathscr{E}_{p}\right\}$ is bounded.

Conversely, suppose that $\sup \left\{|e|: e \in \mathscr{E}_{p}\right\} \leqq k$, for some constant $k$. We use the notation of the proof of [1] Theorem 7.4. Let $\left\{T_{\lambda}\right\}$ be the family of all $p_{\lambda}$-representing operators such that $\left\|T_{\lambda}^{-1}\right\|=1$ for all $\lambda$. Then the set $\left\{\left\|T_{\lambda}\right\|\right\}$ is bounded; for if not, by the proof of [1] Theorem 7.4, there would exist a sequence $\left\{H_{\lambda_{n}}\right\} \subset\left\{H_{\lambda}\right\}$ and elements $x_{n}, y_{n} \in H_{\lambda_{n}}(n=1,2, \cdots)$ such that $\left|f_{y_{n}}-f_{x_{n}}\right| \rightarrow 0$ and $\left|e_{y_{n}}-e_{x_{n}}\right| \rightarrow \infty$, as $n \rightarrow \infty$, which would contradict the fact that $\left|e_{y_{n}}-e_{x_{n}}\right| \leqq 2 k$. It follows now from the proof of [1] Theorem 7.4 that $p$ is uniformly continuous. This completes the proof.

Now let $A$ be a dual $A^{*}$-algebra which is a dense subalgebra of a $B^{*}$-algebra $\mathfrak{A}$, and let $\left\{I_{\lambda}: \lambda \in \Lambda\right\}$ be the family of all minimal closed two-sided ideals of $A$. Clearly each $\mathrm{cl}\left(I_{\lambda}\right)$ is a minimal closed two-sided ideal of $\mathfrak{A}$ and hence *-isomorphic to $L C\left(H_{\lambda}\right)$, for some Hilbert space $H_{\lambda}$. Suppose $q$ is a complementor on $A$ and, for each $\lambda \in \Lambda$, let $q_{\lambda}$ be the complementor on $I_{\lambda}$ induced by $q$. Identifying $I_{\lambda}$ as a subalgebra of $L C\left(H_{\lambda}\right), q_{\lambda}$ induces the complementor $\boldsymbol{p}_{\lambda}$ on $L C\left(H_{\lambda}\right)$ (Lemma 8.5). For each closed right ideal $R_{\lambda}$ of $L C\left(H_{\lambda}\right)$, let $P_{R_{\lambda}}$ be the projection on $R_{\lambda}$ along $R_{\lambda}^{p_{\lambda}}$. Then $P_{R_{\lambda}}$ is a bounded linear operator on $L C\left(H_{\lambda}\right)$ whose operator bound we denote by $\left|P_{R_{\lambda}}\right|$. Let

$$
\begin{aligned}
m_{\lambda} & =\sup \left\{\left|P_{R_{\lambda}}\right|: R_{\lambda} \subset L C\left(H_{\lambda}\right)\right\}, \\
m & =\sup \left\{m_{\lambda}: \lambda \in \Lambda\right\} ;
\end{aligned}
$$

$m$ may be finite or infinite. 
LemMA 8.7. If $I$ is a closed right ideal of $A$, then $I^{q} \cap I_{\lambda}=\left(I \cap I_{\lambda}\right)^{q \lambda}$, for every $\lambda \in A$.

Proof. Since $I \cap I_{\lambda} \subset I$, we have $I^{q} \subset\left(I \cap I_{\lambda}\right)^{q}$ and hence

$$
I^{q} \cap I_{\lambda} \subset\left(I \cap I_{\lambda}\right)^{q \lambda} .
$$

Now, by [1] Lemma 2.1, $\operatorname{cl}\left(I+I_{\lambda}^{q}\right)=I^{q} \cap I_{\lambda}$; hence

$$
\operatorname{cl}\left(I+I_{\lambda}^{q}\right) \cap I_{\lambda}=\left(I^{q} \cap I_{\lambda}\right)^{q} \cap I_{\lambda}=\left(I^{q} \cap I_{\lambda}\right)^{q_{\lambda}} .
$$

Let $x \in\left(I^{q} \cap I_{\lambda}\right)^{q \lambda}$. Then $x \in I_{\lambda}$ and $x=\lim _{n} x_{n}$, where $x_{n}=y_{n}+z_{n}$ with $y_{n} \in I$ and $z_{n} \in I_{\lambda}^{q}(n=1,2, \cdots)$. Since, by [10] Lemma $1, I_{\lambda}^{q}=l\left(I_{\lambda}\right)$ and since $x^{*} \in I_{\lambda}$, we obtain that $x x^{*}=\lim _{n} x_{n} x^{*}=\lim _{n} y_{n} x^{*} \in I$. But, by Theorem 7.1, this means that $x \in I$ and therefore $x \in I \cap I_{\lambda}$. Hence

and consequently

$$
\left(I^{q} \cap I_{\lambda}\right)^{q_{\lambda}} \subset I \cap I_{\lambda}
$$

$$
I^{q} \cap I_{\lambda}=\left(I \cap I_{\lambda}\right)^{q_{\lambda}} .
$$

THEOREM 8.8. Let $A$ be a dual $A^{*}$-algebra which is a dense sub-algebra of a $B^{*}$-algebra $\mathfrak{A}$. Then, for every complementor $q$ on $A$ for which $m$ is finite, the mapping $p: R \rightarrow \operatorname{cl}\left((R \cap A)^{q}\right)$ on the closed right ideals $R$ of $\mathfrak{A}$ is a complementor on $\mathfrak{A}$. If, moreover, $\mathfrak{A}$ has no minimal left ideals of dimension less than three and $p$ is continuous, then there exists an involution ${ }^{* \prime}$ on $\mathfrak{A}$ such that $R^{p}=l(R)^{* \prime}$.

Proof. We use the notation of the paragraph preceding Lemma 8.7. It is clear that $\mathfrak{A}$ is *-isomorphic to $\left(\sum L C\left(H_{\lambda}\right)\right)_{0}$. In what follows we identify $\mathfrak{U}$ with $\left(\sum L C\left(H_{\lambda}\right)\right)_{0}$. Let $q$ be a complementor on $A$ for which $m$ is finite. Let $R$ be a closed right ideal of $\mathfrak{A}$ and, for each $\lambda \in \Lambda$, let $R_{\lambda}=R \cap L C\left(H_{\lambda}\right)$. Then, by [1] Lemma 7.1, $R=\left(\sum R_{\lambda}\right)_{0}$. Define

$$
R^{\prime}=\left(\sum\left[R \cap L C\left(H_{\lambda}\right)\right]^{p_{\lambda}}\right)_{0},
$$

where $\boldsymbol{p}_{\lambda}$ is the complementor on $L C\left(H_{\lambda}\right)$ induced by $q_{\lambda}$. Clearly $R^{\prime}$ is a closed right ideal of $\mathfrak{A}$ and

Hence

$$
R^{\prime} \cap L C\left(H_{\lambda}\right)=R_{\lambda}^{p \lambda}
$$

$$
\left(R^{\prime}\right)^{\prime}=\left(\sum\left[R^{\prime} \cap L C\left(H_{\lambda}\right)\right]^{p_{\lambda}}\right)_{0}=\left(\sum R_{\lambda}\right)_{0}=R .
$$

It is easy to see that the mapping $R \rightarrow R^{\prime}$ has properties $\left(C_{1}\right),\left(C_{3}\right)$ and $\left(C_{4}\right)$. For $x=\left(x_{\lambda}\right) \in \mathfrak{A}$, write $x_{\lambda}=y_{\lambda}+z_{\lambda}, y_{\lambda} \in R_{\lambda}$ and $z_{\lambda} \in R_{\lambda}^{p^{\lambda}}$. We have

$$
\left|y_{\lambda}\right|=\left|P_{R_{\lambda}} x_{\lambda}\right| \leqq m\left|x_{\lambda}\right| \quad(\lambda \in \Lambda) ;
$$

similarly $\left|z_{\lambda}\right| \leqq m\left|x_{\lambda}\right|(\lambda \in \Lambda)$. Hence, since $m$ is finite,

$$
\left(y_{\lambda}\right) \in\left(\sum R_{\lambda}\right)_{0}=R \text { and }\left(z_{\lambda}\right) \in\left(\sum R_{\lambda}^{p_{\lambda}}\right)_{0}=R^{\prime} .
$$

Thus $R+R^{\prime}=\mathfrak{U}$ and consequently $R \rightarrow R^{\prime}$ is a complementor on $\mathfrak{A}$. 
We show next that $R^{\prime}=\operatorname{cl}\left((R \cap A)^{q}\right)=R^{p}$. Let $I=R \cap A$. Since, by [1] Theorem 7.1, we have $\mathrm{cl}\left(I^{q}\right)=\left(\sum\left[\mathrm{cl}\left(I^{q}\right) \cap L C\left(H_{\lambda}\right)\right]\right)_{0}$, it suffices to show that

$$
R_{\lambda}^{p_{\lambda}}=\operatorname{cl}\left(I^{q}\right) \cap L C\left(H_{\lambda}\right)(\lambda \in \Lambda) .
$$

Now, by the duality of $A$, we have

$$
\operatorname{cl}\left(I^{q}\right) \cap I_{\lambda}=\operatorname{cl}\left(I^{q}\right) \cap A \cap I_{\lambda}=I^{q} \cap I_{\lambda} .
$$

Therefore, the duality of $I_{\lambda}$ and Lemma 8.7 give

$$
\begin{aligned}
\operatorname{cl}\left(I^{q}\right) \cap L C\left(H_{\lambda}\right) & =\operatorname{cl}\left(\left[\operatorname{cl}\left(I^{q}\right) \cap L C\left(H_{\lambda}\right)\right] \cap I_{\lambda}\right) \\
& =\operatorname{cl}\left(\operatorname{cl}\left(I^{q}\right) \cap I_{\lambda}\right)=\operatorname{cl}\left(I^{q} \cap I_{\lambda}\right)=\operatorname{cl}\left(\left(I \cap I_{\lambda}\right)^{q \lambda}\right) \\
& =\operatorname{cl}\left(\left(R_{\lambda} \cap I_{\lambda}\right)^{q \lambda}\right)=R_{\lambda}^{p_{\lambda}} .
\end{aligned}
$$

To prove the second part of the theorem, we see that, by [1, Theorem 7.4] and Lemma 8.6, it suffices to show that $\left\{|f|: f \in \mathscr{E}_{p}\right\}$ is bounded. Let $f \in \mathscr{E}_{p}$. Since $f \mathfrak{U} \subset L C\left(H_{\lambda}\right)$, for some $\lambda,|P|_{f \mathfrak{A}} \leqq m$. But $f a=P_{f \mathfrak{a}} a$ for all $a \in \mathfrak{A}$, and so $|f| \leqq m$. This completes the proof of the theorem.

\section{Examples}

As an immediate example of a complemented $A^{*}$-algebra we have an $H^{*}$ algebra (see [10]). We shall now give another example, which we believe has not yet been discussed from this point of view.

Let $H$ be a Hilbert space and $\tau c(H)$ the trace class operators on $H$ with the trace norm $\|\cdot\| \cdot \tau c(H)$ is an $A^{*}$-algebra which is a dense two-sided ideal of $L C(H)$ and, as a Banach space, it is isometrically isomorphic to the conjugate space of $L C(H)$ (see [9] p. 47). Clearly $\tau c(H)$ contains all operators of finite rank as a dense set and hence is an annihilator algebra, in fact it is dual as we shall see.

Now let $\left\{H_{\lambda}: \lambda \in A\right\}$ be a family of Hilbert spaces $H_{\lambda}$ and let $\left(\sum_{\lambda} \tau c\left(H_{\lambda}\right)\right)_{1}$ denote the family of all functions $f$ defined on $A$ such that $f(\lambda) \in \tau c\left(H_{\lambda}\right)$ for each $\lambda$ and such that $\sum_{\lambda}\|f(\lambda)\|<\infty$. It follows that $\left(\sum \tau c\left(H_{\lambda}\right)\right)_{1}$ is a Banach algebra under the norm $\|f\|=\sum_{\lambda}\|f(\lambda)\|$ and the usual operations for functions. $\mathrm{J}_{i}$ is easily verified that, as a Banach space, $\left(\sum \tau c\left(H_{\lambda}\right)\right)_{1}$ is isometrically isomorphic to the conjugate space of $\left(\sum L C\left(H_{\lambda}\right)\right)_{0}$. It is clearly a sub-algebra of $\left(\sum L C\left(H_{\lambda}\right)\right)_{0}$ and an $A^{*}$-algebra under the involution $f \rightarrow f^{*}$, where $f^{*}(\lambda)=f(\lambda)^{* \lambda}(* \lambda$ being the adjoint operation in $\tau c\left(H_{\lambda}\right)$ ).

LeMma 9.1. $\tau c(H)$ is a dual $A^{*}$-algebra and the mapping $I \rightarrow l(I)^{*}$ on the closed right ideals $I$ is a complementor on $\tau c(H)$.

Proof. Let $A=\tau c(H)$ and let $I$ be a closed right ideal of $A$. We show that $\mathscr{J}_{A}\left(\mathscr{S}_{A}(I)\right)=I$. Clearly $I \subset \mathscr{J}_{A}\left(\mathscr{S}_{A}(I)\right)$. Let $T \in \mathscr{J}_{A}\left(\mathscr{S}_{A}(I)\right)$ and $\left\{T_{n}\right\}$ a sequence of operators of finite rank on $H$ such that $\left\|T_{n}-T\right\| \rightarrow 0$ as $n \rightarrow \infty$. Let 
$P$ be the orthogonal projection on $\mathscr{S}_{A}(I)$. Since $P T_{n}$ is finite dimensional with range in $\mathscr{S}_{A}(I)$, [8] Theorem (2.4.18) shows that $P T_{n} \in I$ for all $n=1,2, \cdots$. Clearly $P T=T$. By [9] Lemma 8 , we have

$$
\left\|P T_{n}-T\right\|=\left\|P T_{n}-T P\right\| \leqq|P|\left\|T_{n}-T\right\|,
$$

so that $\left\|P T_{n}-T\right\| \rightarrow 0$ as $n \rightarrow \infty$. Hence $T \in I$ and consequently $\left.\mathscr{J}_{A} \mathscr{S}_{A}(I)\right)=I$. Thus, by [8] Lemma (2.8.24), and the continuity of the involution, $A$ is dual. Let $T \in A$. Then $T=P T+P^{\prime} T$ where $P^{\prime}=1-P$, and, since $P T \in I$ and $P^{\prime} T \in l(I)^{*}$, we have $I+l(I)^{*}=A$. It is now easy to see that the mapping $I \rightarrow l(I)^{*}$ is a complementor on $A$.

THEOREM 9.2. $\left(\sum \tau c\left(H_{\lambda}\right)\right)_{1}$ is a dual $A^{*}$-algebra which is a dense two-sided ideal of $\left(\sum L C\left(H_{\lambda}\right)\right)_{0}$.

PROOF. Let $A=\left(\sum \tau c\left(H_{\lambda}\right)\right)_{1}$ and $\mathfrak{A}=\left(\sum L C\left(H_{\lambda}\right)\right)_{0}$. Identifying $\tau c\left(H_{\lambda}\right)$ as a subalgebra of $A$, we see that $\tau c\left(H_{\lambda}\right)$ is a closed two-sided ideal of $A$ and that $A$ is the direct topological sum of the $\tau c\left(H_{\lambda}\right)$. Therefore, by [8] Theorem (2.8.29), $A$ is an annihilator algebra. Since each $\tau c\left(H_{\lambda}\right)$ is dense in $L C\left(H_{\lambda}\right)$, it is easy to show that $A$ is dense in $\mathfrak{A}$. Moreover, since for all $x_{\lambda} \in L C\left(H_{\lambda}\right)$ and $y_{\lambda} \in \tau c\left(H_{\lambda}\right)$, we have $\left\|x_{\lambda} y_{\lambda}\right\| \leqq\left|x_{\lambda}\right|\left\|y_{\lambda}\right\|$ ([9] Lemma 8, p. 39), it readily follows that $A$ is a twosided ideal of $\mathfrak{A}$. Let $x=\left(x_{\lambda}\right) \in A$. Identifying $\tau c\left(H_{\lambda}\right)$ as a subalgebra of $A$, we have $x \tau c\left(H_{\lambda}\right)=x_{\lambda} \tau c\left(H_{\lambda}\right)$ for all $\lambda$. Therefore, by the duality of $\tau c\left(H_{\lambda}\right), x_{\lambda} \in \operatorname{cl}(x A)$ for all $\lambda$. It is now easy to show that $x \in \operatorname{cl}(x A)$, and so, by Theorem 7.1, $A$ is dual.

COROLLARY 9.3. The mapping $I \rightarrow l(I)^{*}$ on the closed right ideals $I$ of $\left(\sum \tau c\left(H_{\lambda}\right)\right)_{1}$ is a complementor on $\left(\sum \tau c\left(H_{\lambda}\right)\right)_{1}$.

PROOF. This follows from Theorem 9.2 and the proof of Theorem 7.3.

We do not know of an example of a complemented $A^{*}$-algebra which is not a dense two-sided ideal of a $B^{*}$-algebra. Also we do not know if every dual $A^{*}$-algebra is complemented, and conversely if every complimented $A^{*}$-algebra is dual.

\section{References}

[1] F. E. Alexander and B. J. Tomiuk, 'Complemented $B^{*}$-algebras', Trans. Amer. Math. Soc. 137 (1969), 459-480.

[2] F. F. Bonsall and A. W. Goldie, 'Annihilator algebras', Proc. London Math. Soc. (3), 4 (1954), 154-167.

[3] J. Dixmer, $C^{*}$-algèbres et leurs représentations (Gauthier-Villars, Paris, 1964).

[4] I. Kakutani and G. W. Mackey, 'Ring and lattice characterizations of complex Hilbert space', Bull. Amer. Math. Soc. 52 (1964), 727-733.

[5] I. Kaplansky, 'The structure of certain operator algebras', Trans. Amer. Math. Soc. 70 (1951), 219-255.

[6] T. Ogasawara and K. Yoshinaga, 'Weakly completely continuous Banach*-algebras', $J$. Sci. Hiroshima Uni. Ser. A 18 (1954), 15-36. 
[7] T. Ogasawara and K. Yoshinaga, 'A characterization of dual $B^{*}$-algebras', J. Sci. Hiroshima Uni. Ser. A 18 (1954), 179-182.

[8] C. E. Rickart, General theory of Banach algebras (D. Van Nostrand, New York, 1960).

[9] R. Schatten, Norm ideals of completely continuous operators (Springer-Verlag, 1960).

[10] B. J. Tomiuk, 'Structure theory of complemented Banach algebras', Canadian J. of Math. 14 (1962), 651-659.

University of Ottawa

Ottawa, Canada 